\title{
ESTRUTURA DO MICROFITOPLÂNCTON NO SISTEMA ESTUARINO DO RIO GOIANA, PERNAMBUCO, BRASIL
}

Fernando Antônio do Nascimento FEITOSA Maria da Glória Gonçalves da SILVA-CUNHA

José Zanon de Oliveira PASSAVANTE

Sigrid NEUMANN-LEITÃO

Iara Correia LINS

Departamento de Oceanografia da UFPE

\begin{abstract}
RESUMO
Estudos sobre o microfitoplâncton foram realizados no sistema estuarino do rio Goiana, visando conhecer a biodiversidade, sua distribuição espaço-temporal e correlacionar as espécies mais abundantes e freqüentes aos parâmetros hidrológicos. Amostragens foram feitas de março de 1992 a fevereiro de 1993, em quatro estações, durante as baixa-mares e preamares diurnas. As amostras de plâncton foram obtidas com rede padrão de $65 \mu \mathrm{m}$ de abertura de malha. Parâmetros físicos e químicos foram coletados simultaneamente. Foram identificadas 119 espécies de fitoplâncton. O sistema estuarino é polihalino, com variações salinas mais acentuadas no período chuvoso. A associação das unidades amostrais apresentou 3 subgrupos. Os 3 primeiros fatores da ACP explicou $77,83 \%$ da variação dos dados. O estuário é eutrófico, com maiores concentraç̃os de nutrientes no período chuvoso e baixa mar, estando correlacionado inversamente com a produtividade fitoplanctônica.

Palavras chave: Fitoplâncton, Estuário, Associações, Tropical
\end{abstract}

\section{ABSTRACT \\ Microphytoplankton Structure of the Estuarine System of the Riyer Goiana, Pernambuco, Brazil}

Studies about the microphytoplankton were carried out at the estuarine area of the river Goiana to assess the biodiversity, the spatial and temporal distribution., and to correlate the most abundant and frequent species to hydrologicat data. Sampling were carried out from March/1992 to February/1993, in 4 stations at diurnal low and high tides. Plankton collections were made with a standard net, $65 \mu \mathrm{m}$ mesh size. Concurrent physico-chemical data were obtained. One hundred and nineteen phytoplankton species were identified. The ecosystem showed to be polyhaline with higher salinity variations at the tainy season. Samples association presented 3 subgroups. The first 3 PCA explained $77,83 \%$ of the data variance. The estuary is euthrophic and higher nutrients concentrations were found at the rainy season and low tide, inversely correlated to the primary production.

Key words: Phytoplankton, Estuary, Associations, Tropical

\section{INTRODUÇÃO}

Pernambuco abriga cerca de 25.044 hectares de áreas estuarinas (Coelho e Torres, 1982), atualmente bastante reduzidas. Neste cenário natural, destaca-se o sistema estuarino do rio Goiana, localizado ao norte do Estado, por apresentar uma das 
maiores formações de manguezais do litoral, desempenhando um papel fundamental na produtividade do ecossistema, servindo de viveiro natural para inúmeras espécies de invertebrados e vertebrados aquáticos. Além do bosque de mangue, a área caracteriza-se por um verdadeiro meandro de rios, riachos, canais, ilha, ithotas e gamboas $(\mathrm{CPRH}$, 1991).

Apesar da importância deste escossistema, poucos trabalhos de cunho científico foram desenvolvidos na área, o que levou o Departamento de Oceanografia da UFPE a realizar pesquisas preliminares abordando a hidrologia e o plâncton, objetivando diagnosticar as condições ecológicas.

No estudo de plâncton destaca-se presentemente, o do microfitoplâncton, produtor primário da teia trófica pelágica, sendo utilizado intensamente pelas fases larvares de diversos organismos de importância econômica.

A relevância dessa pesquisa consiste em enfocar pela primeira vez a biodiversidade do fitoplâncton no sistema estuarino do rio Goiana, suas distribuições espaço-temporais, correlacionando as espécies mais abundantes e freqüentes aos parâmetros abióticos.

\section{MATERIAL E MÉTODOS}

O sistema estuarino do rio Goiana está situado no extremo norte do Estado de Pernambuco, limite com o Estado da Paraíba (7॰ $32^{\prime}-7^{\circ} 35^{\prime} \mathrm{S}$ e $\left.34^{\circ} 50^{\prime}-34^{\circ} 58^{\prime} \mathrm{W}\right)$. É formado pelos rios Goiana e São Lourenço, localizados inteiramente nele. As margens destes rios delimitam a ilha Tariri, localizada centralmente (Fig. 1).

Nesta área amostras foram coletadas, mensalmente, em quatro estações fixas, no período de março 1992 a fevereiro de 1993, nas baixa-mares e preamares diurnas, em maré de sizígia.

Para o estudo qualitativo do fitoplâncton, as coletas foram feitas, através de arrastos horizontais à superfície com rede de plâncton com abertura de malha dẹ $65 \mu \mathrm{m}$, durante 3 minutos, e logo após fixadas com formol neutro a $4 \%$.

Em laboratório, para cada amostra retirou-se uma sub-amostra de $0,5 \mathrm{ml}$, a qual foi inspecionada em microscópio composto ZEISS, sem prévia oxidação, para identificação das espécies mais frágeis; e outra sub-amostra submetida à oxidação para identificação das espécies de diatomáceas, segundo a técnica de Muller-Melchers \& Ferrando (1956).

.Amostras para análises dos parâmetros físico-químicos foram coletadas com garrafas de Nansen no mesmo local e hora que as amostras de plâncton. Foram obtidos dados da profundidade local através de ecossonda manual (LCD Sounder Plastimo Echotest); de temperatura utilizando um termômetro comum de mercúrio; de salinidade pelo método Mhor-Knudsen, descrito em Strickland \& Parsons (1972); de oxigênio dissolvido pelo método de Winkler, descrito em Strickland \& Parsons (op. cit); da taxa de saturação de oxigênio, segundo a tabela da Unesco (1973); da transparência da água pelo disco de Secchi; do $\mathrm{pH}$ utilizando um pH-metro (Beckman Zeromatic II); da demanda bioquímica do oxigênio (APHA, 1985); de nitrito, nitrato e fosfato, através das técnicas descritas em Strickland \& Parsons (op. cit), do silicato por Grasshoff et al., (1983); e do material em suspensão pelo método de Melo et al ( 1975). 
A análise multivariada dos dados baseou-se no coeficiente de correlação momento-produto de Pearson. A matriz inicial constou de dados de densidade (cel..$^{-1}$ ) dos taxa com freqüência acima que $25 \%$ e dos principais parâmetros abióticos. A matriz foi submetida a uma padronização por fileira para reduzir os efeitos das diferentes escalas e depois aplicou-se a transformação linearizante $\log (x+1)$. A classificação utilizada na associação das amostras foi a aglomerativa hierárquica do "peso proporcional" ( Weighted Pair Group Method Avarage Arithmetics- WPGMA). Foi feita uma análise cofenética para medir a validade dos agrupamentos, cujo valor $>0,8$ é significativo (Rohlf \& Fisher, 1968). Na Análise dos Componentes Principais foi calculado o autovetor e o autovalor. Todos estes cálculos foram feitos utilizando o programa computacional NTSYS (Numerical Taxonomy and Multivariate Analisys System) da Metagraphics Software Corporation, Califórnia - USA.

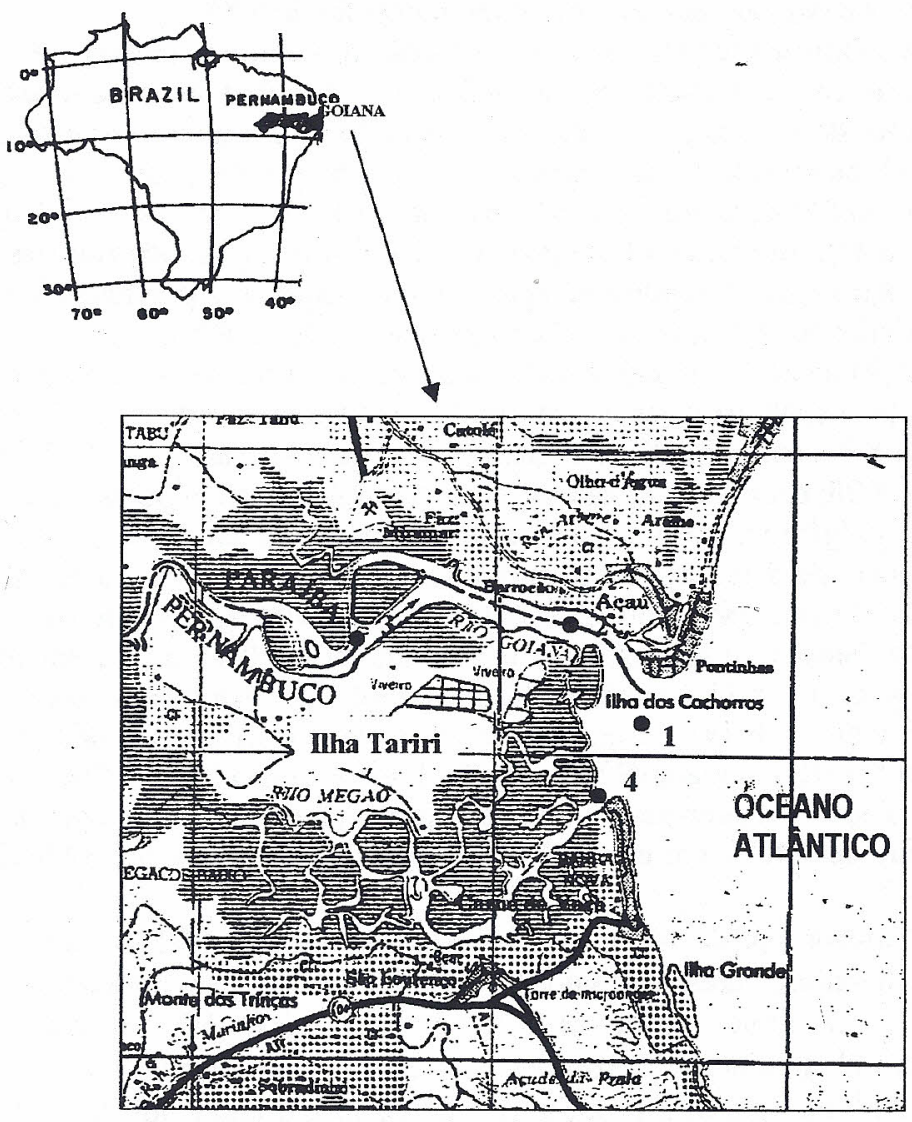

Figura 1 - Área de estudo e estações de coleta no sistema estuarino do rio Goiana, PE (Brasil) 


\section{RESULTADOS}

A tabela 1 apresenta os valores mínimos, máximos e médios dos parâmetros abióticos registrados nas quatro estações estudadas no sistema estuarino do rio Goiana.

A transparência da água apresentou durante as preamares valores mais elevados que as baixa-mares e houve nítida tendência de decrescer das estações 1 e 4, mais na desembocadura, para as estações 2 e 3 mais à montante. Na baixa-mar, o mínimo foi de $0,07 \mathrm{~m}$, no mês de março/92 na estação 3 e o máximo foi de $1,8 \mathrm{~m}$, em novembro/92 na estação 4 e em fevereiro/93 nas estações 1 e 2 . Na preamar, o mínimo foi de $0,1 \mathrm{~m}$, em março/92 na estação 3 , e o máximo foi de $1,8 \mathrm{~m}$, em fevereiro/93 na estação 2.

A temperatura manteve-se mais ou menos estável nas 4 estações. Com relação a variação sazonal, apresentou valores mais baixos nos meses de maio a agosto. $\mathrm{Na}$ baixa-mar o mínimo foi de $23^{\circ} \mathrm{C}$, em julho/92 na estação 4 , e o máximo foi de $29,1^{\circ} \mathrm{C}$ no mês de fevereiro/93, nas estações 2,3 e 4 . Na preamar, o mínimo foi de $24,9^{\circ} \mathrm{C}$, em julho/92 na estação 3 e o máximo foi de $29,8^{\circ} \mathrm{C}$, em fevereiro/93 na estação 2 . A amplitude total em todo período, nas duas marés, foi de $6,8^{\circ} \mathrm{C}$.

A salinidade tendeu a diminuir da desembocadura (estaęões 1 e 4) para a parte mais interna do rio Goiana (estações 2 e 3). Durante as baixa-mares, o mínimo registrado foi de $0,14 \%$ o, em julho/92 na estação 3 , e o máximo foi de $28,72 \%$, em fevereiro/93, na estação 1 . Na preamar, o mínimo foi $0,16 \%$, em março, na estação 3 , e máximo de $36,61 \%$, em dezembro/92 na estação 1 . Essas baixas salinidades registradas no mês de março resultou da forte precipitação pluviométrica ocorrida neste mês $(273,6$ $\mathrm{mm})$. Por outro lado, dezembro/92 apresentou a menor taxa pluviométrica $(10,4 \mathrm{~mm})$ e a maior salinidade. A amplitude de todo período foi de $36,47 \%$ o.

$\mathrm{O} \mathrm{pH}$ da água durante o ciclo anual variou de neutro a alcalino, tendendo mais a alcalino, principalmente nas preamares. $\mathrm{Na}$ baixa-mar, variou de 7,07, em maio/92 na estação 4 a 8,97, em julho/92 na estação 3 . Na preamar, variou de 7,57, em ąbril/92 na estação 2 a 8,38, em março/92 na estação 3 . A amplitude anual foi de 1,9.

$O$ oxigênio dissolvido apresentou variação anual bastante irregular. Houve certa tendência de decrescer da desembocadura para a montante do estuário. Na baixamar, este parâmetro variou de $0,33 \mathrm{ml}^{-1}{ }^{-1}$, em março/92 na estação 3 a $5,15 \mathrm{ml} .1^{-1}$, em maio/92 na estação 1 . Na preamar, o mínimo foi de $0,43 \mathrm{mll}^{-1}$, em março/92, e o máximo foi $6,34 \mathrm{ml} .1^{-1}$, em maio/92 na estação 2. Quanto aos teores de saturação sazonal, durante as baixa-mares, os valores estiveram na sua maioria subsaturados, o mínimo foi 5,75\%, em março/92 na estação 3, e o máximo foi 112,5\%, em agosto/92 na estação 1. Durante a preamar, os valores apresentaram-se, geralmente supersaturados, com mínimo de 7,48\%, em março/92 na estação 3, e máximo de $139,6 \%$, em maio/92 na estação 2.

O menor teor de nitrito foi abaixo de detectável $\left(<0,001 \mu \mathrm{mol} . \mathrm{l}^{-1}\right)$ nas quatro estações, em ambas marés, e o maior foi de $2,410 \mu \mathrm{mol}^{-1^{-1}}$, em março/92, na preamar da estação 3. O nitrato variou de $0,370 \mu \mathrm{mol} . \mathrm{l}^{-1}$ (agosto/92, preamar, estação 4) a $19,950 \mu \mathrm{mol}^{-1} \mathrm{I}^{-1}$ (maio/92, baixa-mar, estação 4). O menor teor de fosfato foi $0,160 \mu$ mol..$^{-1}$, na estação 2 preamar de janeiro/93 e o maior foi de $5,140 \mu \mathrm{mol} . .^{-1}$, na estação 2, baixa-mar de maio/92. O silicato variou de 3,36 $\mu \mathrm{mol}^{1^{-1}}$ em janeiro/93, preamar da estação 1 a 123,060 $\mu \mathrm{mol} . .^{-1} \mathrm{em}$ janeiro/93, na baixa-mar da estação 3 . 
O material em suspensão apresentou maior quantidade na baixa-mar e na estação 3. Nesta maré, o mínimo foi $6,10 \mathrm{mg} \cdot \mathrm{l}^{-1}$, em janeiro/93 na estação 4 , e 0 máximo foi $358 \mathrm{mg} .1^{-1}$, em março/92 na estação 3. Na preamar, o valor mínimo foi 8,10

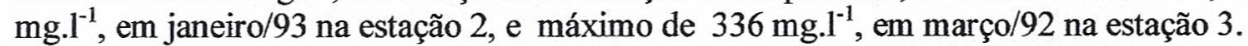

Tabela 1 - Dados hidrológicos mínimos (MÍN), máximos (MÁX) e médios (MÉD) do sistema estuarino do rio Goiana - Pernambuco.

\begin{tabular}{|c|c|c|c|c|c|c|c|c|c|c|c|c|}
\hline \multirow[b]{2}{*}{ PARÂMETROS ABIÓTICOS } & \multicolumn{3}{|c|}{ ESTAÇĀO 1} & \multicolumn{3}{|c|}{ ESTAÇĀO 2} & \multicolumn{3}{|c|}{ ESTAÇÃO 3} & \multicolumn{3}{|c|}{ ESTACCÃO 4} \\
\hline & MiN & MAX & MÉD & MiN & $M A X$ & MÉD & MiN & MÁX & MÉD & MiN & MÁX & MÉD \\
\hline Temperatura da água $\left({ }^{\circ} \mathrm{C}\right)$ & 24,50 & 29,60 & 27,34 & 24,30 & 29,80 & 27,55 & 23,30 & 29,60 & 27,33 & 23,00 & 29,50 & 27,63 \\
\hline & & 36,61 & & & 34,99 & & & 26,65 & & 4,47 & 32,3 & 17,92 \\
\hline & & 8,27 & & & 8,28 & & 7,2 & 8,97 & & 7,07 & 8,13 & 7,47 \\
\hline & 2,82 & 5,75 & 4,54 & 1,20 & 6,34 & 4,15 & 0,33 & 5,89 & 3,50 & 2,66 & 6,50 & 4,37 \\
\hline & 51,93 & 120,96 & 94,05 & 21,31 & 139,65 & 84,48 & 5,7 & 116,40 & 67,47 & 49,35 & 137,71 & 88,45 \\
\hline & & 1,80 & & & 1,80 & & & 1,10 & & 0,30 & 1,80 & 0,76 \\
\hline & 0,001 & 0,910 & 0,195 & 0,000 & 0,580 & 0,221 & 0,000 & 2,410 & 0,361 & 0,000 & 1,040 & 0,205 \\
\hline & 0,900 & 11,670 & 4,112 & 0,700 & 9,140 & 4,708 & 1,310 & 10,880 & 5,509 & 0,370 & 19,950 & 4,609 \\
\hline & 190 & 1,770 & 0,812 & 0,310 & 5,140 & 1,133 & 0,560 & 2,060 & 1,328 & 0,160 & 2,080 & 1,147 \\
\hline Silic & 3,360 & 57,480 & 30,700 & 13,230 & 87,980 & 36,740 & 8,5 & 123,060 & 39,670 & 5,280 & 83,660 & 40,860 \\
\hline n suspensao (mg. ${ }^{\prime}$ ) & 10,60 & 144,40 & 42,23 & 8,10 & 109,60 & 45,83 & 11,30 & 358,00 & 65,10 & 6,10 & 112,00 & 38,23 \\
\hline
\end{tabular}

A clorofila $a$ aumentou da estação 1 para a estação 4, durante as preamares e da estação 1 para a estação 3 , decrescendo na estação 4 durante as baixa-mares. $\mathrm{Na}$ estação 1, o valor médio foi de $10,28 \mathrm{mg} \cdot \mathrm{m}^{-3}$ na baixa-mar e $5,81 \mathrm{mg} \cdot \mathrm{m}^{-3}$ na preamar. $\mathrm{Na}$ estação, 2 o valor médio para a baixa-mar foi de $10,06 \mathrm{mg} \cdot \mathrm{m}^{-3}$ e para a preamar 7,89 mg. $\mathrm{m}^{-3}$. Na estação 3, os valores médios para a baixa-mar e preamar foram $13,55 \mathrm{mg} . \mathrm{m}^{-}$ ${ }_{3}$ e $8,80 \mathrm{mg} \cdot \mathrm{m}^{-3}$, respectivamente. Na estação 4 , o valor médio para a baixa-mar foi de $6,89 \mathrm{mg} \cdot \mathrm{m}^{-3}$ e para a preamar $9,97 \mathrm{mg} \cdot \mathrm{m}^{-3}$.

A produção fitoplanctônica variou de $0,29 \mathrm{mgC} \cdot \mathrm{h}^{-1} \cdot \mathrm{m}^{-3}$ em junho/92 a 196,18 $\mathrm{mgC} \cdot \mathrm{h}^{-1} \cdot \mathrm{m}^{-3}$, em novembro/92, ambas na estação 3 . Os valores médios para cada estação foram Estação 1, 16,67 mgC. $\mathrm{h}^{-1} \cdot \mathrm{m}^{-3}$; estação 2, $13,03 \mathrm{mgC} \cdot \mathrm{h}^{-1} \cdot \mathrm{m}^{-3}$; estação 3, 47,71 $\mathrm{mgC} \cdot \mathrm{h}^{-1} \cdot \mathrm{m}^{-3}$; e estação $4,17,51 \mathrm{mgC} \cdot \mathrm{h}^{-1} \cdot \mathrm{m}^{-3}$.

O microfitoplâncton apresentou alta diversidade taxonômica com 119 espécies (Tab. 2). As diatomáceas totalizaram mais de $75 \%$ em todas as amostras, sendo as espécies mais abundantes Coscinodiscus centralis, Actinoptychus splendens, Thalassiosira sp, Bellerochea malleus e Biddulphia regia. A densidade mínima foi de 40.000 cel..$^{-1}$ na estação 2 e a máxima foi de 10.730 .000 cel. $^{-1}$ na estação 4 (Tab. 3). Destacaram-se as diatomáceas seguidas dos fitoflagelados.

A diversidade específica apresentou valores médios a baixo, com média geral de 2,08 bits.cel $^{-1}$ para a baixa-mar e 2,17 bits.cel $^{-1}$ para a preamar. $O$ valor mínimo registrado foi de 0,71 bits.cel $^{-1}$ na baixa-mar de março/92 na estação 4 e o máximo foi de 3,73 bits.cel $^{-1}$, na preamar de agosto/92, na estação 1 . A eqüitabilidade variou de 0,20 em novembro/92, preamar da estação 3 a 0,88 nas estações 1 (baixa-mar, setembro/92) e 3 (preamar, agosto/92). A média geral para a baixa-mar foi de 0,63 e para a preamar de 0,67 . 
Tabela 2 - Biodiversidade do Microfitoplâncton no sistema estuarino do rio Goiana - Pernambuco, no período de março de 1992 a fevereiro de 1993.

\section{CYANOPHYTA}

Microcystis aenginosa Kutzing

Trichodesmium enythraeum Ehrenberg

Oscillatoria princeps Vaucher ex Gomont

Oscillatoria sp

Spirulina sp

Anabaena Sp

EUGLENOPHYTA

Euglena acus Ehrenberg

Euglena sp

PHYRROPHYTA

Ceratium fusus (Ehrenberg) Dujardin

Protoperidinium sp

Pyrophacus horologicum Stein

Pyrocystis pseudonoctiluca W. Thompson

CHRYSOPHYTA CENTRICAE

Coscinodiscus centralis Ehrenberg

Coscinodiscus excentricus Ehrenberg

Coscinodiscus granii Gough

Coscinodiscus nitidus Gregory

Coscinodiscus oculusiridis Ehrenberg

Coscinodiscus sp

Cyclotella sp

Melosira moniliformis (0. Müller) Agardh

Melosira granulata (Ehrenberg) Ralfs

Paralia sulcata (Ehrenberg) Kutzing

Skeletonema costatum (Greville) Cleve

Thalassiosira gravida Cleve

Thalassiosira subtilis (Ostenfeld) Gran

Thalassiosira sp

Actinoptychus splendens (Shadbolt) Raffs

Actinoptychus undulatus (Bailey) Ralfs

Polymyxus coronalis L. W. Bailey

Rhizosolenia setigera Brightwell

Guinardia stolterfothii H. Peragallo

Rhizosolenia styliformis Brightwell

Actinocyctus $\mathbf{s p}$

Chaetoceros affinis Lauder

Chaetoceros brevis Schutt

Chaetoceros coarctatus Lauder

Chaetoceros curvisetus Cleve

Chaetoceros Iorenzianus Grunow

Chaetoceros mitra (Bailey) Cleve

Chaetoceros perrvianus Ehrenberg

Chaetoceros teres Cleve

Chaetoceros sp

Bellerochea malleus (Brightwell) Van Heurk

Biddulphia aurita (Lyngbye) Brebisson \& Godey

Biddulphia biculphiana Snith

Biddulphia laevis Ehrenberg

Biddulphia Iongicruris Greville

Biddulphia regia (Schultze) Ostenfeld

Cerataulina pelagica (Cleve) Hendey

Cerataulus turgidus Ehrenberg

Ditylum brightwellii (West) Grunow

Hemiaulus hauckii Grunow

Hemiatsus membranaceus Cleve

Hemiaulus $\mathrm{sp}$

Isthmia enervis Ehrenberg

Odontella mobiliensis (Bailey) Grunow

Streptotheca thamensis Shrubsolei

Triceratium aftemans Bailey

Triceratium favus Ehrenberg
Triceratium favis $\mathrm{f}$. quadrata Grunow

Eunotogramma sp

Terpsinoe musica Ehrenberg

Hemidiscus hardmanianus (Greville) Mann

Subclasse: PENNATAE

Asterionellopsis glacialis (Castracane) Round

Climacosphenia moniligera (Lyngbye) Kützing

Fragilaria capucina Desmazières \& Kutzing

Fragilaria sp

Grammatophora angulosa Ehrenberg

Grammatophora marina (Lyngbye) Kützing

Grammatophora oceanica (Ehrenberg) Grunow

Licmophora abbreviata Agardh

Plagiogramma s $p$

Rhabdonema adriaticum Kutaing

Rhabdonema punctatum (Harvey \& Barley) Stodder

Symedra acus Kützing

Simedra ulna (Nitzsch) Ehrenberg

Thalassionema nitzschioides (Grunow) Van Heurck

Thalassiothrix frauenfeldii Grunow

Achnanthes sp

Cocconeis scultellum Ehrenberg

Anomoneis serians (Brébisson) Creve

Caloneis permagna (J. W. Bailey) Cleve

Frickea lewisiana Heiden

Gyrosigma balticum (Ehrenberg) Cleve

Pinnularia nobilis Ehrenberg

Pinnularia clericii var. comentina Frenguelli

Pinnularia stauroptera (Grunow) Rasenhorst

Pleurosigma naviculaceum Brébisson

Pleurosigma sp

Amphora sp

Entomoneis alata (Ehrenberg) Kutzing

Amphiprora paludosa Wm. Smith

Amphiprora sp

Bacillaria paxilffer Gmelin

Cylindrotheca closterium (Ehrenberg) Reimann \& Lewis

Nitzschia obtusa Wm Smith

Nitzschia pungens var. atlantica Cleve

Nitzschia seriata Cleve

Nitzschia sigma (Kutzing) Wm. Smith

Nitzschia socialis Gregory

Nitzschia sp

Campylodiscus clypeus Ehrenberg

Sunirella fastuosa Ehrenberg

Surirella fastuosa var. recendens ( Schmidt) Cleve

Surirella febigenit Lewis

Surirella sp

CHLOROPHYTA

Eudorina elegans Ehrenberg

Eudorina sp

Scenedesmus sp

Ankistrodesmus $\mathbf{S p}$

Closterium $\mathrm{sp}$

Micrasterias borgey f. minor $\mathrm{n}$. var.

Straurastrum lepłocladum Nordstedt

Straurastrum minnesotense Wolle

Straurastrum submanfelditii West

Straurastrum sp

Strourastrum lobatus (Borgesen) Bourrely

Spirogyra oblata Jao

Spirogyra sp 
Tabela3 - Densidade (cel. $\Gamma^{1}$ ) dos grupos fitoplanctônicos no sistema estuarino do rio Goiana Pernambuco.

\begin{tabular}{|c|c|c|c|c|c|c|c|c|}
\hline \multirow[t]{2}{*}{ TAXA } & \multicolumn{2}{|c|}{ ESTAÇÃO 1} & \multicolumn{2}{|c|}{ ESTAÇÃO 2} & \multicolumn{2}{|c|}{ ESTAČ̃O 3} & \multicolumn{2}{|c|}{ ESTAÇÃO 4} \\
\hline & MÍNIMO & MÁXIMO & MINIMO & MÁXIMO & MÍNIMO & MÁXIMO & MINIMO & MAXXIMO \\
\hline Cianoficeas & 0 & 2.000 .000 & 0 & 2.560 .000 & 0 & 1.120 .000 & 0 & 1.300 .000 \\
\hline Euglen & 0 & 100.000 & 0 & 230.000 & 0 & 330.000 & 0 & 140.000 \\
\hline Dinof & 0 & 70.000 & 0 & 700. & 0 & 800 & 0 & 100.000 \\
\hline Diato & 80.000 & 3.960 .000 & 20.000 & 3.260 .000 & 50.000 & 5.400 .000 & 100.000 & 6.750 .000 \\
\hline & 30.000 & 1.100 .000 & 20.000 & 1.500 .000 & 50.000 & 2.160 & 130.000 & 2.300 .000 \\
\hline Cloroficeas & 0 & 110.000 & 0 & 140.000 & 0 & 830.000 & 0 & 140.000 \\
\hline Total & 110000 & 7340000 & 40000 & 8390000 & 100000 & 10640000 & 230000 & 10730000 \\
\hline
\end{tabular}

A associação das amostras apresentou uma correlação cofenética $\mathrm{r}=0,5$, mostrando não haver diferença significativa entre as estações de coleta. Evidenciou-se, contudo, os seguintes subgrupos influenciados pelas marés e períodos sazonais (Fig. 2): Subgrupo 1: associou os meses com maior intensidade pluviométrica de 1992, e as baixa-mares de todas as estações.

Subgrupo 2: associou os meses com menor intensidade pluviométrica de 1992 com as preamares destes meses.

Subgrupo 3: associou os meses com menor intensidade pluviométrica de $1993 \mathrm{com}$ as preamares destes meses.

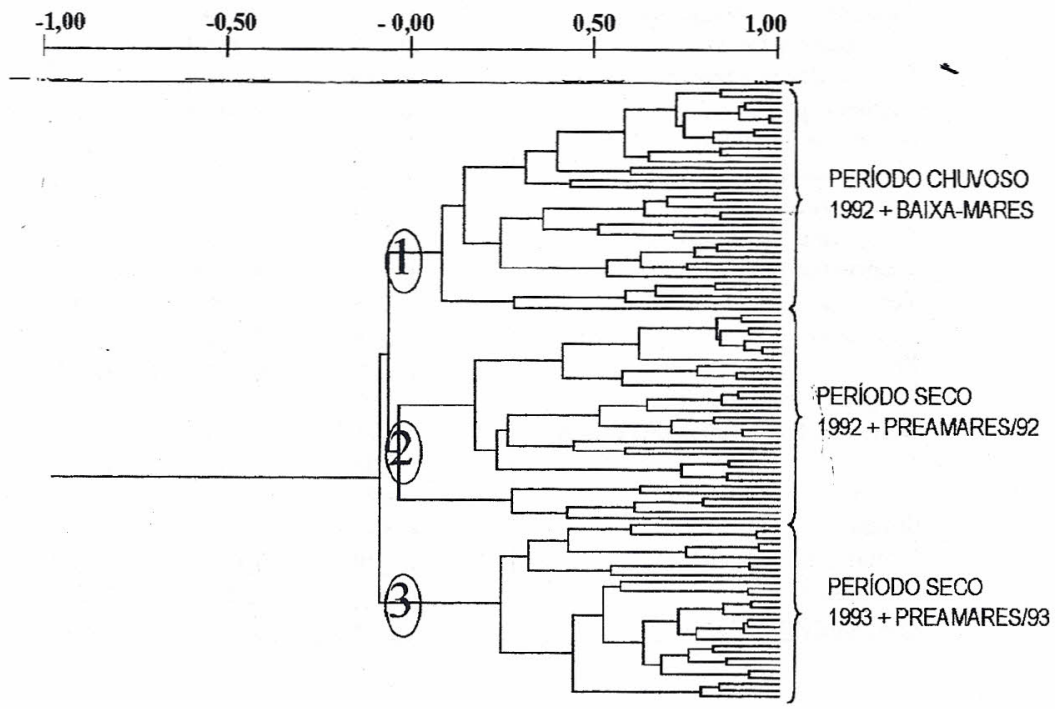

Figura 2 - Dendrograma da associação de amostras no sistema estuarino do rio Goiana - PE, no período de março de 1992 a fevereiro de 1993.

Trab. Oceanog. Univ. Fed. PE, Recife, 27(2): 15-25 , 1999. 
A análise dos componentes principais, com base nas espécies mais freqüentes e parâmetros ambientais, mostrou que os três primeiros fatores explicaram $77,83 \%$ das variações ocorridas no sistema estuarino do rio Goiana. O Fator 1 explicou $34,17 \%$ da variação dos dados e relacionando as variáveis transparência e oxigênio dissolvido diretamente com Bacillaria paxillifer, Nitzschia sigma e inversamente com Chaetoceros lorenzianus, pH, DBO material em suspensão, clorofila a e a produtividade primária. O Fator 2 explicou $23,34 \%$ da variação dos dados e associou positivamente Biddulphia regia e Protoperidinium sp com a temperatura e salinidade, e todos estes correlacionados inversamente com Chaetoceros teres, Coscinodiscus centralis, nitrato e fosfato. O Fator 3 explicou $20,32 \%$ da variação dos dados e associou positivamente Gyrosigma balticum e Pyrophacus horologicum ao silicato e estes associados inversamente a Bellerochea malleus (Tab. 4).

Tabela 4 - Análise dos Componentes Principais dos taxa fitoplanctônicos e parâmetros ambientais no sistema estuarino do rio Goiana - Pernambuco.

\begin{tabular}{l|r|r|r}
\hline \multicolumn{1}{c|}{ TAXA E PARAMETROS } & $\begin{array}{r}\text { FATOR 1 } \\
(34,17 \%)\end{array}$ & $\begin{array}{r}\text { FATOR 2 } \\
(23,34 \%)\end{array}$ & $\begin{array}{r}\text { FATOR 3 } \\
(20,32 \%)\end{array}$ \\
\hline Actinoptychus splendens & 0,092 & 0,531 & $-0,313$ \\
Asterionellopsis glacialis & $-0,372$ & 0,078 & 0,589 \\
Bacillaria paxillifer & $\underline{0,863}$ & 0,389 & 0,153 \\
Bellerochea malleus & 0,221 & 0,453 & $\underline{-0,841}$ \\
Biddulphia mobiliensis & $-0,117$ & 0,234 & $-0,147$ \\
Biddulphia regia & $-0,047$ & $\underline{0,718}$ & 0,050 \\
Chaetoceros affins & 0,447 & 0,248 & 0,302 \\
Chaetoceros curvisetus & $-0,475$ & 0,072 & $-0,621$ \\
Chaetoceros lorenziamus & $\underline{-0,892}$ & 0,279 & $-0,495$ \\
Chaetoceros teres & 0,632 & $-0,990$ & $-0,312$ \\
Coscinodiscus centralis & $-0,318$ & $-0,751$ & 0,300 \\
Euglena sp & $-0,172$ & $-0,141$ & $-0,265$ \\
Gyrosigma balticum & 0,503 & 0,347 & $\underline{0,702}$ \\
Nitzchia sigma & $\underline{0,853}$ & 0,134 & 0,080 \\
Protoperidinium sp & 0,129 & $\underline{0,705}$ & 0,605 \\
Pyrophacus horologicum & 0,252 & 0,284 & $\underline{0,855}$ \\
Transparência & $\underline{0,919}$ & 0,038 & $-0,524$ \\
Temperatura & 0,695 & 0,699 & $-0,144$ \\
Salinidade & 0,329 & $\underline{0,831}$ & $-0,119$ \\
PH & $\underline{-0,829}$ & $-0,013$ & $-0,253$ \\
Oxigênio dissolvido & $\underline{0,896}$ & 0,264 & 0,482 \\
Demanda Bioquímica de Oxigênio & $-0,611$ & 0,293 & 0,331 \\
Nitrato & 0,203 & $-0,600$ & $-0,177$ \\
Fosfato & 0,696 & $-0,741$ & $-0,129$ \\
Silicato & $-0,368$ & $-0,022$ & $\underline{0,925}$ \\
Material em suspensão & $\underline{-0,898}$ & $-0,101$ & 0,038 \\
Clorofila a & $\underline{-0,887}$ & 0,389 & $-0,100$ \\
Produção primária & $\underline{-0,759}$ & 0,181 & 0,614 \\
\hline
\end{tabular}




\section{DISCUSSÃO}

O sistema estuarino do rio Goiana apresentou uma mistura intensa dos fluxos marinho e limnético, com predomínio do marinho, podendo ser classificado como homogêneo (tipo $\mathrm{C}$ ) no período seco e moderadamente estratificado no período chuvoso (tipo B) de acordo com o sistema esquematizado por Tundisi (1970). Segundo a classificação das águas, apresentada no Simpósio de Veneza, em 1959, o estuário teve um regime predominantemente polialino. Esta maior influência salina no sistema estuarino do rio Goiana se faz sentir até as partes mais internas (estação 3), onde nas preamares do período seco chega a alcançar $25 \%$, sendo fundamental na renovação da águas poluídas à montante do rio.

Nestas áreas mais internas do rio Goiana, o oxigênio dissolvido apresentou baixas taxas de saturação, principalmente durante o período seco e baixa-mares, elevando-se para valores saturados ou mesmo supersaturados nas proximidades da desembocadura. Com base nas taxas de saturação de oxigênio, Macêdo \& Costa (1978) criaram um sistema de classificação para os estuários do nordeste do Brasil, estando o do rio Goiana enquadrado nas áreas mais à montante em zona poluída a semi-poluída mudando para zona de baixa saturação à medida que se aproxima da sua desembocadura, alcançando eventualmente zona supersaturada, nas preamares, livre de poluição.

Esta melhor qualidade da água próximo à desembocadura se deve à forte influência marinha, que traz para o sistema estuarino a comunidade fitoplanctônica costeira, sendo as diatomáceas o grupo dominante. É comum o predomínio de diatomáceas nos estuários, correspondendo a $77 \%$ da comunidade no sistema estuarino do rio Goiana. A maioria destas espécies são marinhas neríticas eurialinas, onde em outros estuários de Pernambuco chegam a dominar com mais de $80 \%$ da comunidade (Passavante,1979; Eskinazi-Leça et al., 1980; Silva-Cunha et al., 1987/89; Feitosa, 1988; Barros-Franca \& Batista, 1991; Lacerda, 1994; Eskinazi-Leça e Koening, 1995/96; Flores Montes, 1996; Koening, 1997).

A Análise dos Componentes Principais indicou que os parâmetros bióticos e abióticos analisados explicaram grande parte das variações ocorridas no sistema estuarino do rio Goiana, demonstrando que as espécies e fatores com maior papel ecológico no ambiente são Bacillaria paxillifer, Nitzschia sigma relacionadas diretamente ao fluxo marinho (maior transparência e $\mathrm{O}_{2}$ ) e Chaetoceros lorenzianus, $\mathrm{pH}, \mathrm{DBO}$, material em suspensão, clorofila $a$ e a produtividade primária associados ao fluxo limnético e ao do extensivo manguezal existente na área. $O$ predomínio de Nitzschia sigma e Bacillaria paxillifer espécies bentônicas indica pouca profundidade local e intensa dinâmica das marés.

Papel ecológico importante, também, tiveram as diatomáceas marinhas eurialinas Biddulphia regia e Coscinodiscus centralis em conjunto com Chaetoceros lorenzianus, embora sejam de origem marinha, por serem generalistas se adaptaram bem às condições do sistema estuarino do rio Goiana, aproveitando de forma ótima os nutrientes.

A associação das amostras do sistema estuarino do rio Goiana indicaram que, para a maioria dos parâmetros ambientais, as diferenças entre os períodos seco e chuvoso e entre as preamares e baixa-mares não foram muito acentuadas, sendo o 
sistema comandado em grande parte pelo fluxo marinho. Diferença espacial maior fói observada para a biomassa fitoplanctônica, com alta produção nas áreas mais internas do estuário, decrescendo em direção à desembocadura. Esta alta biomassa em termos de clorofila $a$ é resultante do florescimento de poucas espécies generalistas (restrategistas) como Biddulphia regia, Coscinodiscus centralis, Gyrosigma balticum e Bellerochea malleus, dentre outras, gerando uma baixa diversidade de espécies nesta área.

A baixa diversidade de espécies e a alta produtividade permitem caracterizar o sistema estuarino do rio Goiana como eutrofizado, estando sob a influência de vários estressores, principalmente da agroindústria açucareira, tendo o fluxo marinho papel importante como minimizador dos impactos.

\section{REFERÊNCIAS BIBLIOGRÁFICAS}

APHA. 1985. Standard methods for the examination of waste-water. 16 ed. New York, $1268 p$.

Barros-Franca, L. \& Batista, R. N. 1991. Distribuição e abundância relativa do fitoplâncton no complexo estuarino lagunar de Suape. Anais do 4 Encontro Brasileiro de Plâncton, Recife. p. 97-113.

Coelho, P. A. \& Torres, M. F. A. 1982. Áreas estuarinas de Pernambuco. Trabalhos Oceanográficos Universidade Federal de Pernambuco, Recife, 17: 67-80.

CPRH. 1997. Companhia Pernambucana para o Controle da Poluição e Recursos Hídricos. Qualidade das águas da Bacia do rio Goiana 1986-1989. Série Publicações Técnicas, Recife, 8, 20p.

Eskinazi-Leça, E. \& Koening, M. L. 1986. Distribuição das diatomáceas (Bacillariophyceae) na área de Suape (Pernambuco - Brasil). Trabalhos Oceanográficos Universidade Federal de Pernambuco, Recife, 19:73 - 100.

Eskinazi-Leça, E., Macêdo, S. J. \& Passavante, J. Z. O. 1980. Estudo ecológico da região de Itamaracá, Pernambuco, Brasil. V. Composição e distribuição do microfitoplâncton na região do Canal de Santa Cruz. Trabalhos Oceanográficos Universidade Federal Pernambuco, Recife, 15: 185-262.

Feitosa, F. A. N. 1988. Produção primária do fitoplâncton correlacionada com parâmetros bióticos e abióticos na Bacia do Pina (Recife - Pernambuco, Brasil). Recife, 270p: Dissertação de Mestrado, Departamento de Oceanografia, Centro de Tecnologia, Universidade Federal de Pernambuco.

Flores Montes, M. J. 1996. Variação nictemeral do fitoplâncton e parâmetros hidrológicos no Canal de Santa Cruz, Itamaracá, PE, Recife, 199p. Dissertação de Mestrado. Universidade Federal de Pernambuco.

Grasshoff, K., Ehrhardt, M. \& Kremling, K. (Ed.). 1983. Methods of Seawater Analysis. Verlag Chemie, $2^{a}$ edição. 419 p.

Koening, M. L. 1997. Ecologia e dinâmica do fitoplâncton no estuário do rio Ipojuca, após a implantação do porto de Suape (PE - Brasil). Recife - PE 263p. Tese de Doutorado, Departamento de Botânica, Universidade Federal Rural de Pernambuco. 
Lacerda, S. R. 1994. Variação diurna e sazonal do fitoplâncton do estuário do rio Paripe (Itamaracá - Pernambuco - Brasil). Recife. 146p. Dissertação de Mestrado, Departamento de Oceanografia, Universidade Federal de Pernambuco.

Macêdo, S. J. \& Costa, K. M. P. 1978. Estudo ecológico da região de Itamaracá Pernambuco - Brasil. Condições hidrológicas do estuário do Rio Botafogo. Ciência e Cultura, 30 (7): 346-368.

Muller-Melchers, F. C. \& Fernando, H. J. 1957. Plankton Diatoms of the "TokoMaru" voyage (Brazil Coast). Boletim do Instituto Oceanográfico de São Paulo, São Paulo, 8 (1-2): 111-38.

Passavante, J. Z. O. 1979. Produção Primária do Fitoplâncton do Canal de Santa Cruz (Itamaracá). São Paulo, 188p. Tese de Doutorado, Universidade de São Paulo.

Rohlf, F. J. \& Fisher, D. L. 1968. Teste for hierarchical structure in random data sets. Systematic Zoology, 17:407-412.

Silva-Cunha, M. G. G., Eskinazi-Leça, E. \& Almeida, C. D. P. 1987/89. Taxonomia e ecologia do microzooplâncton do estuário do rio Timbó (Pernambuco - Brasil). Trabalhos Oceanográficos Universidade Federal de Pernambuco, Recife, 20:35-52.

Strickland, J. D. H. \& Parsons, T. R. 1972. A pratical handbook of seawater analysis. Bulletin Fiheries Research Board of Canada, Ottawa, 167:207-211.

Tundisi, J. G. 1970. O plâncton estuarino. Contribuições avulsas do Instituto Oceanográfico, São Paulo, série Oceanografia Biológica, 19: 1-22.

UNESCO. 1973. International Oceanographic Table. Opeat Britain. Wormly, 2, 141p. 